www.jmscr.igmpublication.org Impact Factor 5.244

Index Copernicus Value: 83.27 ISSN (e)-2347-176x ISSN (p) 2455-0450 crossref DOI:_http://dx.doi.org/10.18535/jmscr/v4i8.40

\title{
Impact of BCG Vaccination Status on Sputum Conversion Rate in New Sputum Positive cases of Pulmonary Tuberculosis
}

\author{
Authors
}

\section{Priyam Goswami ${ }^{1}$, Basanta Laskar ${ }^{2}$, Jayanta Kumar Nath $^{3}$}

${ }^{1}$ Assistant Professor of Medicine, Dept of Medicine, Assam Medical College and Hospital,

Dibrugarh, Assam India

${ }^{2}$ Professor of Medicine, Dept of TB \& Chest Diseases, AMCH, Dibrugarh, Assam, India

${ }^{3}$ Post Graduate Trainee, Dept of Medicine, Assam Medical College and Hospital, Dibrugarh, Assam. India

Email:priyam014@rediffmail.com,drblaskar@gmail.com,jayantanath69@gmail.com

\begin{abstract}
Objectives: Sputum conversion rates $(S C R)$ at the end of intensive phase of treatment of pulmonary tuberculosis varies and knowledge of factors involved is, inadequate. It has been reported that presence of a BCG scar was associated with greater rate of sputum conversion at the end of intensive phase (IP) of treatment. Hence, this study was undertaken with aim of studying the relationship between $B C G$ vaccination status and sputum conversion rate in pulmonary tuberculosis (PTB) patients.

Materials And Methods: This was a hospital based observational study done at Assam Medical College and Hospital, Dibrugarh from July 2012 to June 2013. All new sputum smear positive (SSP-PTB) patients were put on antitubercular therapy under DOTS and followed up with repeat sputum microscopy at the end of intensive phase. A comparative study on the outcome was done between BCG scar positive and negative cases and statistical analysis done.
\end{abstract}

Results: All the 102 new SSP-PTB cases successfully completed intensive phase treatment under DOTS. Out of these 19 patients had to be put on extended IP of 1 month. BCG scar was present in $64.17 \%$ cases and absent in $35.29 \%$. Overall, $81.37 \%$ of new sputum positive (NSP) cases converted at the end of second month IP on DOTS. In BCG scar present group, sputum conversion rate (SCR) was $90.90 \%$ whereas in scar absent group it was $63.89 \%$, showing a significant ' $p$ ' value of 0.0013.In the extended IP group $94.48 \%$ cases with scar converted, while $22.22 \%$ without BCG scar remained non-converted with a significant ' $p$ ' value of 0.0009 .

Conclusion: New SSP-PTB patients with evidence of previous BCG vaccination, show a significantly better sputum conversion rate at the end of intensive phase of DOTS.

Keywords: Pulmonary tuberculosis, BCG vaccine, Sputum conversion rate.

\section{Introduction}

Tuberculosis remains one of the major public health problems worldwide, particularly in the developing countries. India has the highest TB burden of the world accounting for one-fifth of global incidence. ${ }^{2}$
In India approximately 1.8 million cases are sputum smear positive (SSP) and responsible for transmission of disease. Early detection and effective treatment of SSP-PTB cases can cut down the chain of transmission. ${ }^{3}$ WHO emphasizes the 
necessity of monitoring treatment by bacteriologic follow up examination in SSP-PTB patients. Sputum examination results at the end of two months intensive phase of treatment determines the next therapeutic regimen whether to go for continuation or extended intensive phase ${ }^{2,3}$. Several studies have shown that persistent sputum positivity at the end of two months of ATT can predict adverse outcomes in terms of increased failure and/or relapse rates ${ }^{4,5}$. Knowledge of factors associated with persistent sputum positivity at the end of two months of ATT may therefore be useful to the clinician to manage their patients better and improve outcomes

Several previous studies have identified factors such as pretreatment high sputum acid-fast bacilli (AFB) smear grading, gender, smoking, age, multiple cavitary lesions and low BMI as potential predictors of low sputum conversion ${ }^{4,6-10}$.

Interestingly, it has been reported that the presence of a BCG scar was significantly associated with greater rate of sputum conversion at the end of intensive phase. ${ }^{1}$ Hence, this study was undertaken with the objective of finding the BCG vaccination status of sputum positive patients on DOTS therapy under RNTCP and to study the relationship between $\mathrm{BCG}$ vaccination status and sputum conversion rate at the end of intensive phase (IP).

\section{Materials and Methods}

The present study was conducted in Assam Medical College and Hospital, Dibrugarh from July 2012 to June 2013.Permission was taken from the institutional ethics committee before carrying out the study.A total of 102 cases greater than 13 years of age and diagnosed as new smear positive PTB patients at DOTS Centre, Assam Medical College and Hospital and DTC, Dibrugarh attached to the department of TB \& Chest Diseases were included in the study. Patients with known chronic liver disease, pregnant women, malignancy or immunosuppression, terminal illness were excluded from the study. Informed consent was taken from each patient.
All patients were subjected to a thorough clinical examination and data recorded in a prepared proforma. All 102 cases were enrolled in the first 9 months of study and another 3 months were kept for follow up. The results of pre-treatment sputum smear examination with Zeihl-Neelsen technique (2 samples) were recorded .All patients were put on DOTS therapy category I under RNTCP. Documentation of BCG scar for each NSP patient was done after examination of left upper arm. Presence of BCG scar was regarded as evidence of BCG vaccination. Patients were followed up with repeat sputum microscopy at 2 months. In 19 cases intensive phase had to be extended by another month according to RNTCP guidelines. Routine laboratory investigations were also done.

The data collected were analysed and statistical study done.

\section{Results}

All the 102 new SSP-PTB patients successfully completed intensive phase treatment under DOTS. Out of these 19 patients had to be put on extended IP for 1 month. There was a male preponderance of cases $(59.80 \%)$ and maximum patients were in the age group of $13-24$ yrs $(37.25 \%)$.Weight loss was the most common symptom $(80.39 \%)$ followed by cough $(73.53 \%)$ and fever $(70.59 \%)$.

Table 1: Presenting symptoms

\begin{tabular}{|l|l|l|}
\hline SYMPTOMS & NUMBER (n) & PERCENTAGE (\%) \\
\hline $\begin{array}{l}\text { Weight Loss/Loss of } \\
\text { Appetite }\end{array}$ & 82 & 80.39 \\
\hline Cough & 75 & 73.53 \\
\hline Fever & 72 & 70.59 \\
\hline Dyspnoea & 38 & 37.25 \\
\hline Night Sweats & 35 & 34.31 \\
\hline Fatigue & 29 & 28.43 \\
\hline Chest Pain & 24 & 23.53 \\
\hline Haemoptysis & 10 & 9.80 \\
\hline
\end{tabular}

BCG scar was present in $64.71 \%$ cases and absent in $35.29 \%$.In older age groups the non-vaccinated cases were more and highest between 13-25 years. It was observed that in more than half of the patients $(53.92 \%)$ pretreatment sputum smear grading was high $(3+)$ and maximum were in the age group of 35-44 years. 
Table-2 : Relationship of BCG scar with sputum microscopy after 2 months of IP

\begin{tabular}{|l|l|l|l|l|l|l|l|l|}
\hline \multirow{2}{*}{$\begin{array}{l}\text { Age Group } \\
\text { (Yrs) }\end{array}$} & \multicolumn{2}{|l|}{ Bcg Scar Present } & \multicolumn{3}{l|}{ Bcg Scar Absent } \\
\cline { 2 - 9 } & \multicolumn{2}{|l|}{$\begin{array}{l}\text { Pretreatmen } \\
\text { t cases }\end{array}$} & \multicolumn{2}{l|}{$\begin{array}{l}\text { Conversion } \\
\text { after } \\
\text { months }\end{array}$} & \multicolumn{2}{l|}{$\begin{array}{l}\text { Pretreatment } \\
\text { cases }\end{array}$} & \multicolumn{2}{l|}{$\begin{array}{l}\text { Conversion } \\
\text { after 2 months }\end{array}$} \\
\cline { 2 - 9 } & $\mathrm{n}$ & $(\%)$ & $\mathrm{n}$ & $(\%)$ & $\mathrm{n}$ & $(\%)$ & $\mathrm{n}$ & $(\%)$ \\
\hline $13-24$ & 30 & 45.45 & 26 & 86.61 & 8 & 22.22 & 4 & 50.0 \\
\hline $25-34$ & 21 & 31.82 & 19 & 90.47 & 8 & 22.22 & 5 & 62.5 \\
\hline $35-44$ & 11 & 16.67 & 11 & 100.0 & 7 & 19.44 & 4 & 57.14 \\
\hline$\geq 45$ & 4 & 6.06 & 4 & 100.0 & 13 & 36.11 & 10 & 76.9 \\
\hline TOTAL & 66 & 100 & 60 & 90.90 & 36 & 100 & 23 & 63.89 \\
\hline
\end{tabular}

'p' value -0.0013

It was seen that at the end of 2 months IP SCR was $81.37 \%$ in all NSP cases. In the BCG scar present group SCR was $90.90 \%$ whereas in scar absent group it was $63.89 \%$, showing a significant ' $p$ ' value of 0.0013 as shown it Table-2.

On evaluating data of conversion and nonconversion at the end of extended IP, it showed that $98.48 \%$ cases with scar converted, while $22.22 \%$ cases without scar did not convert having a significant ' $p$ ' value of 0.0009 (Table-3). In the initial high sputum grade (3+),BCG scar present group had $84.85 \%$ conversion whereas scar absent group had $45.45 \%$ conversion after 2 months of IP. A total of 9 cases $(8.82 \%)$ remained positive even after extended IP, of which 1 case $(11.11 \%)$ had a scar and $8(88.89 \%)$ was without a scar.

Table-3: Showing BCG scar with sputum conversion rate after extended IP

\begin{tabular}{|l|l|l|l|l|l|l|l|l|}
\hline \multirow{2}{*}{$\begin{array}{l}\text { Age } \\
\text { Group }\end{array}$} & \multicolumn{3}{|l|}{ BCG Scar Present } \\
\cline { 2 - 9 } & $\begin{array}{l}\text { Pretreat } \\
\text { Ment } \\
\text { Cases }\end{array}$ & $\begin{array}{l}\text { Conversion } \\
\text { after } \\
\text { Extended } \\
\text { IP }\end{array}$ & \multicolumn{2}{l|}{$\begin{array}{l}\text { Pretreat } \\
\text { Ment Cases }\end{array}$} & \multicolumn{2}{l|}{$\begin{array}{l}\text { Conversion } \\
\text { After } \\
\text { Extended IP }\end{array}$} \\
\cline { 2 - 9 } & $\mathrm{n}$ & $(\%)$ & $\mathrm{n}$ & $(\%)$ & $\mathrm{n}$ & $(\%)$ & $\mathrm{n}$ & $(\%)$ \\
\hline $13-24$ & 30 & 45.45 & 30 & 100 & 8 & 22.22 & 5 & 62.50 \\
\hline $25-34$ & 21 & 31.82 & 20 & 95. & 8 & 22.22 & 7 & 87.50 \\
\hline $35-44$ & 11 & 16.67 & 11 & 100 & 7 & 19.44 & 5 & 71.43 \\
\hline$\geq 45$ & 4 & 6.06 & 4 & 100 & 13 & 36.11 & 11 & 84.62 \\
\hline Total & 66 & 100 & 65 & 98. & 36 & 100 & 28 & 77.78 \\
& & & 48 & & & & \\
\hline
\end{tabular}

Hence analysis of data showed a significant association between BCG scar and sputum smear conversion after both 2months IP and end of extended IP.

\section{Discussion}

Tuberculosis remains as a leading cause of morbidity and mortality all over the world. A total of 102 cases of new sputum smear positive cases were studied.

In the present study most of the cases were observed between $13-24$ years $(37.25 \%)$ followed by the next group of 25-34 years (28.83\%). In the first group male and female had equal incidence, but on advancement of ages male preponderance was noted. Age incidence in this study is closely related to similar study by Hans L Rider et al(1985) where highest incidence was observed between 25-29 years ${ }^{4}$. In another study by Tanzanian and British Medical Research Council in 1985 most of the patients were in the age group of 25-34 years ${ }^{11}$.

In this study $59.8 \%$ cases were male and $40.2 \%$ cases female, the male female ratio being 1.48:1. Tanzanian and BMRC study also found a male preponderance with $64 \%$ males versus $36 \%$ females ${ }^{11}$.Marcelo $\mathrm{F}$ Rabhi et al also found male preponderance which was similar to the present study ${ }^{12}$.Current smokers were observed to be the most affected (53.92\%) than past smokers and nonsmokers.C Kuaban, R Barme et al 2012 in their study observed that tobacco use was associated with persistent sputum positivity ${ }^{5}$.

In the present study, BCG scar was present in $64.7 \%$ of cases of which $60.66 \%$ were male and $70.73 \%$ cases were female. $78.95 \%$ patients in the 13-24 years age group were the highest vaccinated and the group of 45 years and above were the lowest vaccinated (25.53\%). Colditz GA, Brewer TE et al (1994) 13 stated that BCG vaccine significantly reduces the risk of $\mathrm{TB}$ by $50 \%$ and age at vaccination did not enhance predictiveness of BCG efficacy. The present study showed similar results with Colditz et al.

Sputum conversion rate in PTB patients with adequate drug compliance is a potential indicator of treatment outcome. Non-conversion may lead to failure, default or drug resistance. In this study 
overall SCR after 2 months of DOTS was $81.37 \%$ which increased to $90.10 \%$ after extended IP. The results are similar to that of the official report of District Tuberculosis Office, Dibrugarh. SCR for 2011 was $82 \%$ and $91 \%$ at end of 2 months and extended IP, and for the year 2012 it was $80 \%$ and $92 \%$ respectively. It was also found that $95.74 \%$ with low smear grade converted while only $69.09 \%$ cases of pre-treatment high sputum smear grade converted showing a significant 'p' value of 0.0006 . Tiwari et al 2012, at National Tuberculosis Institute observed that SCR at the end of 2months IP was $57.9 \%$ in high positive grade and $71.6 \%$ among low positive group. After extended IP of 1month SCR was $85.2 \%$ and $92.3 \%$ respectively.

In this present study BCG scar present group showed $90.90 \%$ and $98.48 \%$ sputum conversion at the end of 2months and end of extended IP, in comparision to conversion of $63.80 \%$ and $77.77 \%$ in scar absent group. The protective effect provided by BCG vaccine as observed by different studies is very much heterogenous. Several trials show efficacy ranging from 0 to $80 \%$. But studies showing BCG vaccination status and SCR are rare. In a study conducted by Kidolah Jeremiah et al on 754 culture positive PTB patients in 2006-2008 in Tanzania, it was shown that the presence of a BCG scar was found to be associated with a $43 \%$ lower risk of persistence of sputum smear positivity at the second month after initiation of anti-TB treatment. Moreover they also opined that PTB patients in absence of a BCG scar, male sex, high initial AFB sputum grading and low BMI are at risk of remaining culture and or sputum smear positive at the second month of ATT.

\section{Conclusion}

In conclusion, the new smear positive pulmonary tuberculosis patients with evidence of previous BCG vaccination, show a significantly better sputum conversion rate at the end of intensive phase of DOTS as well as the end of extended IP. Pretreatment high smear grade cases without a BCG scar are found at significant risk of non-conversion at the end of IP. However, study in a large number of cases over a wider geographical area need to be undertaken before coming to a conclusion in this respect.

\section{Acknowledgement}

We acknowledge the valuable contribution made by late Dr.D.N. Bhattacharjee towards this study.

\section{References}

1. Kidola Jeremiah, George Pray God Daniel Faurholt-Jepsen et al: BCG vaccination status may predict sputum conversion in patients with pulmonary tuberculosis; a new consideration of old vaccine BMJI Journal, Issue 1, 2011

2. WHO Global TB Report,2010 Update

3. World Health Organization, Treatment of Tuberculosis, guidelines for national programme, Fourth Edition, 2010, WHO

4. Rieder, H.L. Sputum smear conversion during directly observed treatment for Tuberculosis. Tubercle. Lung. Dis. 1996; 77: 124-129.

5. C.Kuaban, R.Bame, L. Mouangue, S.Djella and C. Yomgni on East African Medical Journal Vol.86 No.5 May 2009

6. Sterne JA, Rodrigues LC, Guedes IN. Does the efficacy of BCG decline with time since vaccination? Int $\mathrm{J}$ Tuber Lung Dis. 1998;2:200-7

7. Telzak EE ,Fazal BA, Pollard CL et al: factors influencing sputum smear and culture conversion time among patients with new case pulmonary tuberculosis.

8. Gular M, Unsal E, Dursun B, et al. factors influencing sputum smear and culture conversion time among patients with new case pulmonary tuberculosis. Int J Clin Pract 2007;61:231-5.

9. Gopi PG, Chandrasekaran V, Subramani R, et al. Association of conversion and cure with initial smear grading among new smearcase pulmonary tuberculosis patients treated with category 1 regimen. India $\mathrm{J}$ Med Res 2006;123:807-14. 
10. Banu Rekha VV. Balasubramanian R, Swaminathan S, et al. Sputum conversion at the end of intensive phase of category-1 regimen in the treatment of pulmonary tuberculosis patients with diabetes mellitus or HIV infection: an analysis of risk factors. Indian J Med Res 2007;126:452-8

11. Tanzanian/BMRC collective study 1985 , Tuberculosis in Tanzania -A national survey of newly notified cases. Tubercle 66:161

12. Marcelo fouad Rabahi, Adriana Batista Rodriquez, Fernanda queiroz De Mello, Joaquim Caetano de Almeida Netto Afranio Lineo, Kritski, Clementito, Filho University, Federal University of Rio De Janerio, RJ, 1998.

13. Colditz GA, Brewer, TF, Berkey CS, Wilson ME, Burdick E, Fineberg HV, et al. Efficacy of BCG vaccine in the prevention of tuberculosis. Meta-analysis of the published literature. JAMA. 1994;271:698-702.

14. Tiwari S, Kumar ,Kapoor SK (2012) :National Tuberculosis Institute (DBT); Relation of SCR and pretreatment sputum smearing grading.

15. Comstock GW, Edwards PQ. An American view of BCG vaccination, illustrated by results of a controlled trial in Puerto Rico. Scand J Resp Dis. 1972;53:207-17. 